\title{
The UK Midwifery Study System: investigating uncommon events and conditions in midwifery-led settings
}

\section{Authors}

Rachel Rowe, Jennifer J Kurinczuk, Jennifer Hollowell, Mervi Jokinen \& Marian Knight, on behalf of UKMidSS

Developed in consultation with the Royal College of Midwives, consultant midwives and others, the UK Midwifery Study System (UKMidSS) is a new reporting and research system to investigate uncommon events and conditions in midwifery-led settings. Funded by the National Institute for Health Research, it is co-ordinated at the National Perinatal Epidemiology Unit (NPEU) in Oxford. All alongside midwifery units (AMUs) in the UK have been invited to join this collaboration, with the aim that from January 2016 they will all be involved in reporting to UKMidSS.

There is good evidence about the benefits of care in midwifery-led settings during labour and birth for healthy women with straightforward pregnancies (Birthplace in England Collaborative Group, 2011, Hollowell et al, 2011, Hodnett et al, 2012, National Institute for Health and Care Excellence, 2014) and increasing numbers of women are planning birth in midwifery units (National Audit Office, 2013). Current national guidelines recommend that women may choose any birth setting, but that for healthy women with straightforward pregnancies midwifery units are "particularly suitable". (National Institute for Health and Care Excellence, 2014). This presents an opportunity to further develop the evidence base regarding care in midwifery-led settings.

Since 2005, the UK Obstetric Surveillance System (UKOSS) has been used to identify and study uncommon events, outcomes and disorders of pregnancy, including 'near-miss' events, in all consultant-led obstetric units in the UK (Knight and Lindquist, 2013). Using similar methods, UKMidSS will provide valuable evidence on uncommon events and conditions in midwifery-led settings. This will include investigating outcomes for women at 'higher risk' of complications who 
British Journal of Midwifery: final authors approved version

nevertheless plan birth in a midwifery unit and estimating incidence and risk factors for a range of adverse outcomes, some of which may be 'near-miss' events or quality of care indicators, providing evidence to guide midwifery-led care in the future.

In the first instance UKMidSS will cover AMUs only, because currently the large majority of births in midwifery-led settings are planned and take place in AMUs. In the future, with additional resources, we hope to expand to include freestanding midwifery units and planned home births.

\section{How will UKMidSS work?}

UKMidSS will use a similar monthly notification system to that used by UKOSS, but instead of monthly notification cards and paper data collection forms, we will use email and web-based data collection. In every AMU in the UK, we will identify one or two UKMidSS notifying midwives. Every month these UKMidSS notifiers will receive an email asking them to inform the UKMidSS coordinating centre at the NPEU of the number of women with the condition(s) or event(s) being studied who were admitted for labour care in their unit in the previous month. If there have been no women with the condition(s) or event(s) being studied, they will be asked to make a 'nil report'. Clicking on a link provided in this email will enable the information to be sent directly to the NPEU. 'Denominator data' on the number of women admitted for labour care and giving birth in each unit will also be collected. This will be particularly important as there are no routine sources of this information and without it we will not be able to reliably estimate prevalence or incidence that is the proportion of women planning AMU birth affected by the condition or event.

Based on the experience of UKOSS we anticipate that UKMidSS notifiers will use a range of different methods to identify women with the relevant condition or event in their unit. These might include discussion at regular meetings, emailing colleagues, checking manual and electronic data systems or a simple list on a board in the unit. 
British Journal of Midwifery: final authors approved version

When a woman with the condition is notified to the NPEU a second email will be generated with a link to a secure web-based data collection form to collect more detailed information. These forms will be developed specifically for each condition or event being studied and designed to be quick and easy to complete, using only information recorded in the woman's notes. Only anonymised information will be collected; no names, addresses, postcodes, dates of birth, hospital or NHS numbers will ever be requested. UKMidSS notifiers will be asked to keep their own record linking women's names with UKMidSS case IDs so they can retrieve women's notes when needed, for example to check apparent discrepancies in data submitted.

For some studies UKMidSS will also need to collect information about controls or comparison women. UKMidSS notifiers will be asked to identify suitable controls, for example the two women admitted for labour care in the unit immediately prior to the case, and complete data collection forms for these women as well. UKMidSS has received ethical approval from the National Research Ethics Service Committee South West - Frenchay (REC ref. 15/SW/0166) to collect anonymised data about cases and controls/comparison women in this way without consent from individual women.

\section{What conditions and events will be studied?}

The first two UKMidSS studies will investigate: (i) outcomes in severely obese women (with a body mass index $>35 \mathrm{~kg} / \mathrm{m}^{2}$ ) admitted for labour in an AMU, in the first year, and (ii) incidence and risk factors associated with admission to a neonatal unit for babies born in an AMU, in the second year. Each study will collect information for one year.

Maternal obesity is a recognised risk factor for complications and adverse outcomes of pregnancy, labour and birth (Yu et al, 2006, Heslehurst et al, 2008). National guidance recommends that severely obese women should plan birth in an OU (National Institute for Health and Care Excellence, 2014), but in 2008-10 around $1 \%$ of women planning birth in AMUs were severely obese (Hollowell et al, 2011) and this may be increasing. Research on women planning birth in OUs indicates that otherwise healthy obese multiparous women may have lower intrapartum-related risks than was 
British Journal of Midwifery: final authors approved version

previously thought,(Hollowell et al, 2013) and suggests that some severely obese women may safely be managed in an AMU. This national cohort study will estimate the proportion of women planning AMU birth who are severely obese, and describe their labour management, complications and outcomes compared with women of normal weight planning AMU birth.

The second UKMidSS study will investigate women whose baby is admitted to a neonatal unit after birth in an AMU. In the Birthplace cohort study, around $40 \%$ of the adverse perinatal outcomes in births planned in midwifery-led settings occurred in births which took place in the original setting, that is where no transfer took place.(Hollowell et al, 2011) Neonatal admission following birth in an $\mathrm{AMU}$ is therefore a potential indicator of a 'near-miss' event where different management might have made a difference to outcome. This study will estimate the proportion of AMU births where the baby is admitted to a neonatal unit and investigate risk factors, including women's clinical and socio-demographic characteristics that may be associated with outcome in these cases.

\section{UKMidSS: an ongoing national collaboration}

UKMidSS will be a national collaboration between midwives and researchers across the UK. The participation of all eligible units, with complete and reliable case notification and data collection, will ensure that the results of UKMidSS are generalisable and therefore useful to all practicing midwives. Results of studies will be reported promptly via the Twitter handle @NPEU_UKMidSS, the UKMidSS website (www.npeu.ox.ac.uk/ukmidss), quarterly newsletters, annual reports, in peer reviewed publications and at conferences.

Suggestions for future UKMidSS studies are welcome from researchers and midwives, and will be considered by the UKMidSS Steering Group, which is made up of senior midwives from England, Wales, Scotland and Northern Ireland, an obstetrician, a neonatologist, lay members, researchers and representatives from the Royal College of Midwives and Royal College of Obstetricians and Gynaecologists. We hope that by establishing this infrastructure, UKMidSS will contribute to the 
British Journal of Midwifery: final authors approved version

development of the evidence base for midwifery-led care and to research capacity development in midwifery.

\section{References}

Birthplace in England Collaborative Group (2011). Perinatal and maternal outcomes by planned place of birth for healthy women with low risk pregnancies: the Birthplace in England national prospective cohort study. BMJ 343: d7400.

Heslehurst N, Simpson H, Ells L, Rankin J, Wilkinson J, Lang R, Brown TJ and Summerbell CD (2008). The impact of maternal BMI status on pregnancy outcomes with immediate short-term obstetric resource implications: a meta-analysis. Obesity Reviews 9(6): 635-683.

Hodnett ED, Downe S and Walsh D (2012). Alternative versus conventional institutional settings for birth. Cochrane Database Syst Rev(8): Art. No.: CD000012. DOI:

000010.001002/14651858.CD14000012.pub14651854.

Hollowell J, Pillas D, Rowe R, Linsell L, Knight M and Brocklehurst P (2013). The impact of maternal obesity on intrapartum outcomes in otherwise low risk women: secondary analysis of the Birthplace national prospective cohort study. BJOG: An International Journal of Obstetrics \& Gynaecology.

Hollowell J, Puddicombe D, Rowe R, Linsell L, Hardy P, Stewart M, Newburn M, McCourt C, Sandall J, Macfarlane A, Silverton L and Brocklehurst P (2011). The Birthplace national prospective cohort study: perinatal and maternal outcomes by planned place of birth. Birthplace in England research programme. Final report part 4. London, NIHR Service Delivery and Organisation programme.

Knight M and Lindquist A (2013). The UK Obstetric Surveillance System: Impact on patient safety. Best Practice \& Research Clinical Obstetrics \& Gynaecology 27(4): 621-630.

National Audit Office (2013). Maternity services in England. HC 794, Session 2013-14. London, The Stationery Office. 
British Journal of Midwifery: final authors approved version

National Institute for Health and Care Excellence (2014). Intrapartum Care: Care of Healthy Women and their Babies During Childbirth. NICE guideline CG190. London.

Yu CKH, Teoh TG and Robinson S (2006). Review article: Obesity in pregnancy. British Journal of Obstetrics and Gynaecology 113(10): 1117-1125. 